\title{
Compuestos oligoméricos para láminas delgadas. Estudio de sus propiedades electrónicas por espectroscopía Raman
}

\author{
J. CASADO ${ }^{1}$, H. MUGURUMA ${ }^{2}$, S. HOTTA ${ }^{3}$, V. HERNÁNDEZ ${ }^{1}$ Y J. T. LÓPEZ NAVARRETE ${ }^{1}$ \\ ${ }^{1}$ Departamento de Química Física, Universidad de Málaga, 29071-Málaga, Spain. \\ ${ }^{2}$ Department of Environmental Systems Engineering, Kochi University of Technology, Tosayamada, Kochi 782-8502, Japan. \\ ${ }^{3}$ Matsushita Research Institute Tokyo, Inc. Advanced Materials Research Laboratory, 3-10-1 Higashimita, Tama-ku, Kawasaki 214-8501, Japan.
}

En este artículo analizamos los espectros Raman de dos oligómeros de tiofeno funcionalizados selectivamente con grupos aminometilos en sus posiciones $\alpha, \alpha^{\prime}$ tanto en estado neutro como oxidado. Los resultados obtenidos pueden ser utilizados para analizar las propiedades electrónicas de dichos compuestos cuando se disponen en láminas delgadas de tipo LangmuirBlodgett o CVD.

Palabras Clave: Espectroscopía Raman, oligotiofenos, oxidación, láminas delgadas.

Oligomeric compounds for thin films. Raman study of their electronic properties

In this paper we analyse the infrared and Raman spectra of two thiophene-based oligomers functionalized with aminomethyl groups in the $\alpha, \alpha^{\prime}$-positions, in both neutral and oxidized states. These results will be extrapoled when these systems are assembled in Langmuir-Blodgett or CVD thin films.

Keywords: Raman spectroscopy, oligothiophenes, oxidation, thin films.

\section{INTRODUCCIÓN}

Actualmente una de las alternativas más prometedoras a los semiconductores inorgánicos ( $\mathrm{Si}, \mathrm{Ge}$, etc.) la constituyen sus homólogos de base orgánica. Dentro de éstos se han desarrollado dos grandes grupos, las denominadas sales orgánicas de transferencia de carga, entre las que destacan los derivados de TTF-TCNQ(1) (tetratiofulvaleno tetracianoquinodimetano), y los polímeros conductores.

Los polímeros conductores se han utilizado en un gran número de aplicaciones que van desde la mimetización de tejidos vivos como músculos o nervios(2), a la utilización como sistemas conductores de la electricidad(3) (poliacetileno dopado), sistemas electrocrómicos(4), electroluminiscentes(5), fotoconductores(6), etc. Sin embargo, y desde un punto de vista estructural, los polímeros conductores presentan el problema intrínseco a los propios métodos de síntesis de la distribución ancha de pesos moleculares y de longitudes de conjugación. Esto hace que el conocimiento y optimización de las propiedades tanto estructurales como electrónicas se vuelva de difícil solución. Para evitar este problema, un gran número de laboratorios se han dedicado al estudio de sistemas oligoméricos. Con estas nuevas moléculas se puede establecer de una forma precisa y exacta la relación entre la estructura o funcionalización del sistema y las propiedades físicas; ésto permite no sólo optimizar dichas propiedades sino proponer estructuras a la carta que satisfagan las necesidades tecnológicas requeridas.

La utilización en muchos campos de la tecnología, caso de la microelectrónica, de láminas delgadas de espesor controla- do (del orden de $\AA$ ) hace que se hayan desarrollado diferentes técnicas de obtención de las mismas y entre las que destacan las técnicas de Langmuir-Blodgett o técnicas de deposición controlada de vapor(7).

El objetivo de este trabajo se centra en el análisis de cómo se alteran las propiedades electrónicas de una serie de oligómeros conjugados, específicamente funcionalizados en sus posiciones $\alpha$-terminales, para su uso como láminas delgadas. Analizaremos si la unión al sustrato por estas posiciones altera las propiedades conjugacionales del sistema tanto en sus estados neutro como oxidado. Para llevar a cabo nuestros estudios utilizaremos la espectroscopía Raman, principalmente. Esta técnica, como se describe en la bibliografía, nos permite el análisis tanto de las longitudes de conjugación en estado neutro como de los defectos conjugacionales o de carga responsables de los mecanismos microscópicos que originan gran parte de las propiedades y aplicaciones mencionadas.

\section{PROCEDIMIENTO EXPERIMENTAL}

Los oligómeros estudiados en este trabajo son dos aminometiloligotiofenos (ver esquema 1), el dicloruro de $\alpha, \alpha^{\prime}-$ bisamoniometiltetratiófeno (Cl-BAMQtT) y el dicloruro de $\alpha, \alpha^{\prime}$-bisamoniometilpentatiófeno (Cl-BAMQqT). Ambos compuestos han sido obtenidos mediante esquemas de reacción previamente publicados(8). La oxidación de las muestras se ha llevado a cabo utilizando vapores de iodo. La utilización de iodo como agente oxidante nos garantiza que la oxidación 
se produce sobre la estructura $\pi$-electrónica del sistema, sin ataque alguno sobre los grupos aminometilénicos finales(9). Dicha reacción se muestra en el esquema 2. Los espectros Raman se registraron a temperatura ambiente usando un espectrómetro Raman Bruker Equinox 55 modelo FRA / 106-S. La fuente excitatriz utilizada fue la línea a $1064 \mathrm{~nm}$ proveniente de un láser de Nd-YAG, mientras que la detección de la señal vibracional se obtuvo con un detector de Germanio previamente enfriado a la temperatura del $\mathrm{N}_{2}$ líquido. La radiación Raman dispersada se recogió con una configuración óptica en back-scattering acumulando en todos los casos 1000 registros con una resolución espectral de $4 \mathrm{~cm}^{-1}$. Las muestras sólidas se introdujeron en capilares sellados de vidrio, una vez situadas en la cámara de muestra, focalizamos la radiación láser sobre la muestra manteniendo una potencia de radiación de $20 \mathrm{~mW}$ para las muestras oxidadas y de $60 \mathrm{~mW}$ para las neutras.

\section{RESULTADOS Y DISCUSIÓN}

\subsection{Materiales en Estado Neutro}

Los espectros Raman de ambos oligómeros en estado neutro se muestran en las Figuras 1 y 2 junto a los espectros Raman del correspondiente oligotiofeno de la misma longitud pero con las posiciones $\alpha$-terminales sustituidas con grupos metilo, DMQtT y DMQqT, respectivamente. La metilación de las posiciones $\alpha$ y $\alpha^{\prime}$ no altera la conjugación electrónica del oligotiofeno como ya se ha mostrado anteriormente(10). Esto nos permite utilizar los espectros Raman del $\alpha, \alpha^{\prime}$-dimetiltetratiofeno y del $\alpha$, $\alpha^{\prime}$-dimetilpentatiofeno como sistemas referencia en los cuales la extensión de las interacciones $\pi$-electrónicas es máxima.

Además de la funcionalización específica del sistema, la cual determina fundamentalmente la conjugación electrónica y cuyo análisis es el objetivo de este trabajo, otro factor que influye en la deslocalización molecular es la conformación. En este sentido, y aunque no disponemos de resultados de difracción de rayos $\mathrm{X}$, podemos suponer que en estado sólido ambos compuestos presentan una disposición coplanar de los anillos con los átomos de azufre en posiciones anti. Esta aproximación se basa en la comparación de los espectros infrarrojos y Raman de cada oligómero y la comprobación de que se verifica el principio de exclusión mutua para el sistema con centro de inversión, es decir, para el Cl-BAMQtT, lo cual es consecuencia directa de la existencia de una estructura coplanar.

En el análisis de las especies neutras, centraremos nuestra atención en dos bandas: la denominada línea A que aparece entre 1550 y $1520 \mathrm{~cm}^{-1}$ y que se puede asignar a una vibración de tensión simétrica de los dobles enlaces situados, fundamentalmente, en los anillos terminales, $v_{\mathrm{S}} \mathrm{C}=\mathrm{C}$; y la llamada línea $\mathrm{B}$ que aparece alrededor de $1480 \mathrm{~cm}^{-1} \mathrm{y}$ que se puede describir como una vibración de tensión simétrica de los dobles enlaces de los anillos internos(10). Estas dos vibraciones contienen la denominada coordenada de conjugación efectiva, dicha vibración define y da cuenta del estado de conjugación del sistema y de sus posibles alteraciones(11).

Si nos fijamos en el espectro Raman de Cl-BAMQtT y Cl-BAMQqT en estado neutro, las líneas A y B aparecen a 1536 $\mathrm{cm}^{-1}$ y $1476 \mathrm{~cm}^{-1}$, y a $1523 \mathrm{~cm}^{-1}$ y $1476 \mathrm{~cm}^{-1}$, respectivamente; es decir, a frecuencias similares a las de sus homólogos dimetilados. Por otra parte, el perfil espectral de intensidades en ambas series de compuestos es similar. Podemos decir que en oligotiofenos con el mismo número de anillos, la extensión de la conjugación electrónica en estado neutro es equivalente.<smiles>CCc1ccc(-c2ccc(-c3ccc(-c4ccc(CCN)s4)s3)s2)s1</smiles>

\section{Cl-BAMQtT}

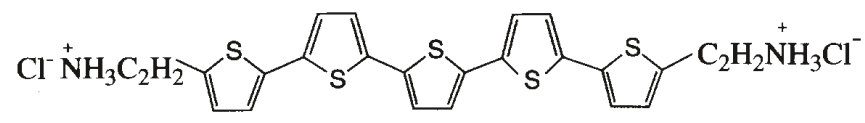

\section{Cl-BAMQqT}

Esquema 1. Estructura química de los oligómeros estudiados en este trabajo.

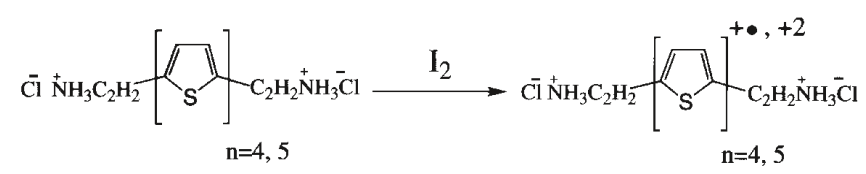

Esquema 2. Reacción de oxidación de Cl-BAMQtT y Cl-BAMQqT con iodo.

\subsection{Materiales en Estado Oxidado}

Tras la oxidación de los materiales neutros generamos estructuras de tipo quinoide en los anillos de tiofeno inicialmente aromáticos. Según predice la teoría de la Coordenada de la Conjugación Efectiva (11) las bandas vibracionales involucradas en la conjugación electrónica en estado neutro sufren una relajación a menores frecuencias. Como podemos observar en las Figuras 3 y 4, donde mostramos los espectros Raman de Cl-BAMQtT y Cl-BAMQqT oxidados con iodo, comprobamos que las bandas más importantes de los espectros aparecen alrededor de $1415 \mathrm{~cm}^{-1}$, lo que está de acuerdo con las predicciones teóricas. Si nuevamente comparamos los espectros Raman de tetrámero y pentámero oxidados con iodo, con los de sus homólogos dimetilados y oxidados podemos confirmar que, tanto las frecuencias como el perfil espectral de intensidades, son esencialmente los mismos.

\section{CONCLUSIONES}

Del análisis comparativo de los espectros FT-Raman de Cl-BAMQtT y Cl-BAMQqT con los de sus homólogos dimetilados podemos concluir que, tanto en estado neutro como oxidado, el aumento de la procesabilidad de estos oligómeros debido a la introducción de grupos amonio en las posiciones terminales no modifica significativamente las propiedades conjugacionales del núcleo de anillos de tiofeno. Como consecuencia, dichos compuestos son buenos candidatos para ser utilizados en láminas delgadas ya que tanto el procesado en disolución como la unión química o física a substratos soporte no parece alterar las propiedades electrónicas. 


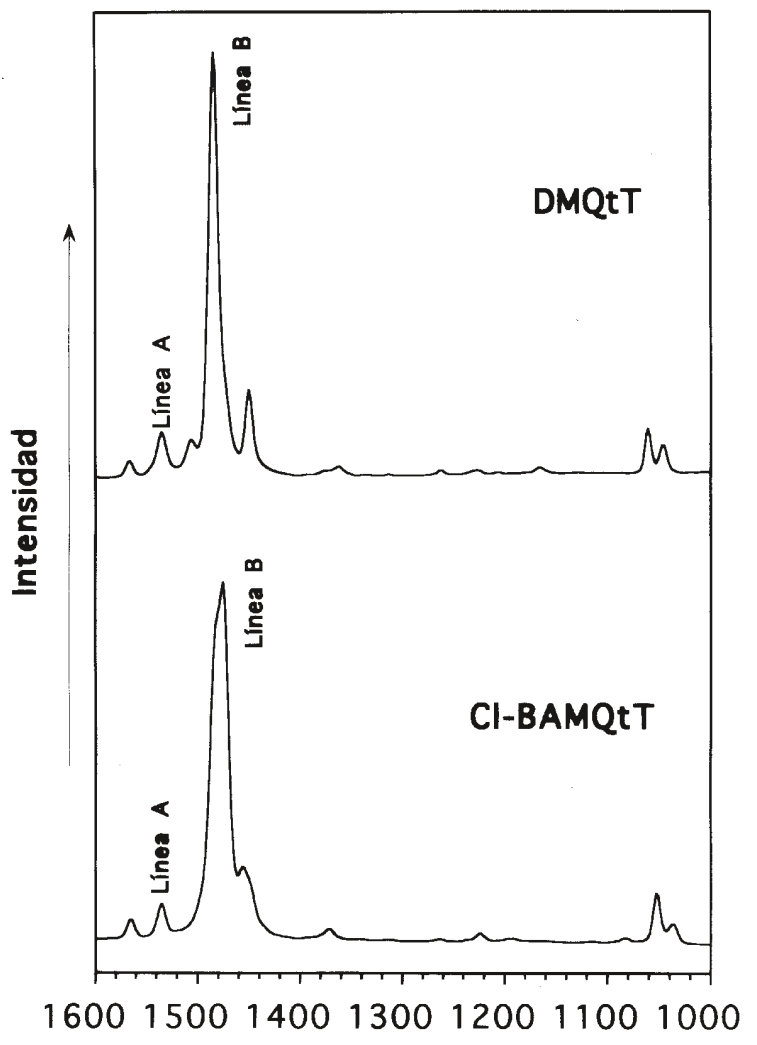

Frecuencia $/ \mathrm{cm}^{-1}$

Figura 1. Espectros Raman de DMQtT ( $\alpha, \alpha^{\prime}$-dimetiltetratiofeno) y Cl-BAMQtT en estado neutro.

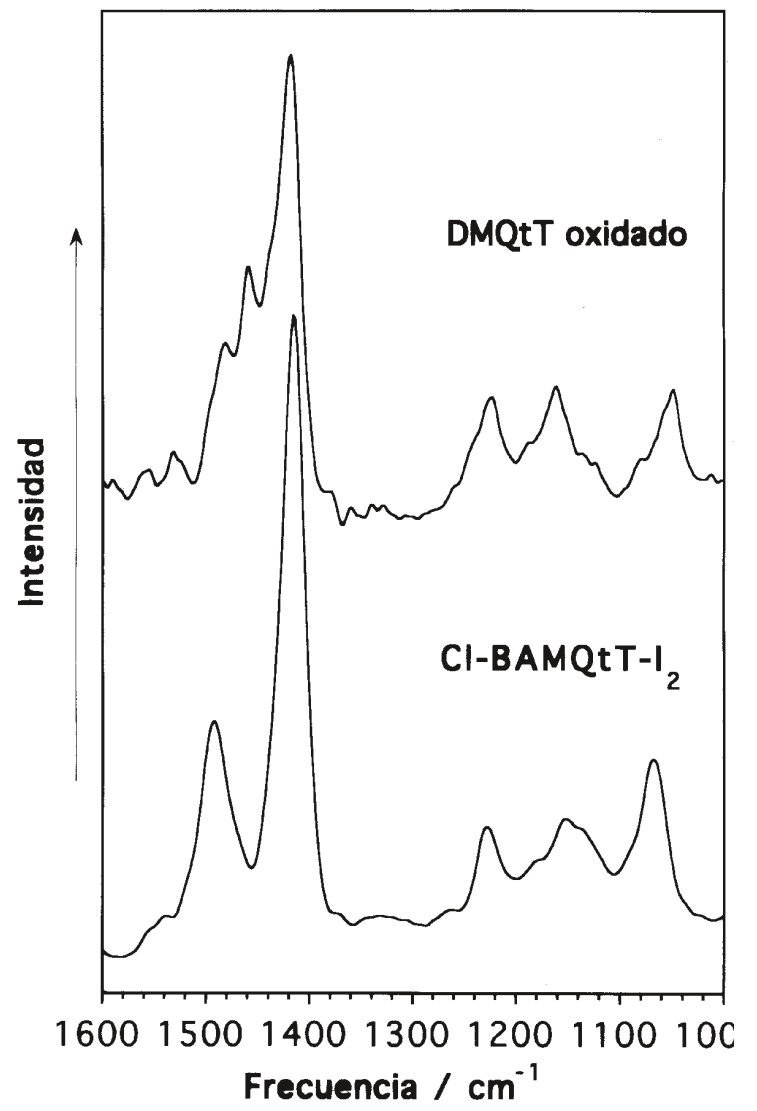

Figura 3. Espectros Raman de DMQtT y de Cl-BAMQtT oxidados con iodo.

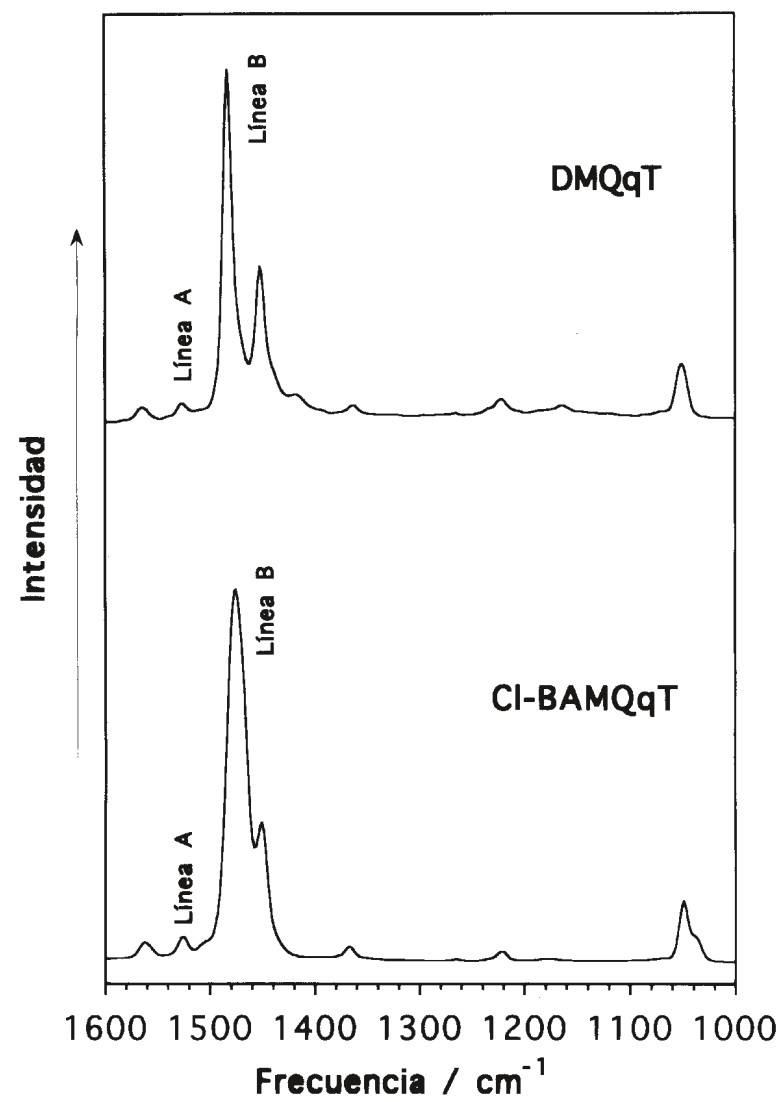

Figura 2. Espectros Raman de DMQqT ( $\alpha, \alpha^{\prime}$-dimetilpentatiofeno) y Cl-BAMQqT en estado neutro.

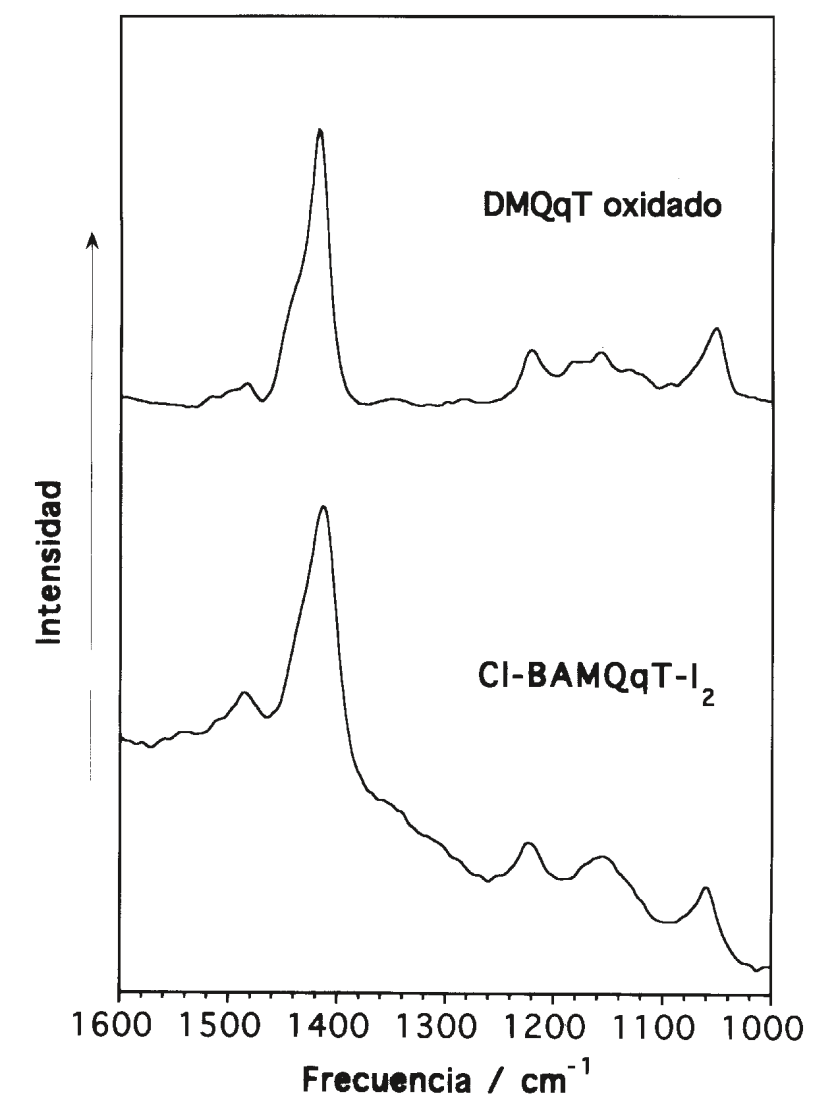

Figura 4. Espectros Raman de DMQqT y de Cl-BAMQqT oxidados con iodo. 


\section{AGRADECIMIENTOS}

J. C. agradece al Ministerio de Educación y Cultura la concesión de una beca predoctoral de Formación de Personal Investigador, sector de Formación de Profesorado Universitario.

Los autores desean expresar su agradecimiento al MEC por el apoyo económico prestado a través de los proyectos PB960682 y IFD97-C03.

\section{BIBLIOGRAFÍA}

1. J. Ferraris, D. O. Cowan, V. J. Walatka, J. M. Perltein. J. Am. Chem. Soc. 95, 948 (1973).

2. T. F. Otero. "Conducting Polymers, Electrochemistry and Biomimicking Processes, Modern Aspects of Electrochemistry", Plenum Press, vol. 33, 1999.
3. H. Shirakawa, E. J. Louis, A. G. MacDiarmid, C. K. Chiang y A. J. Heeger. J. Chem. Soc . Chem. Commun. 578 (1977).

4. M. Pyo y J. R. Reynolds. Chemistry of Materials 8, 128 (1996).

5. A. Dodabalapur, L. Torsi, H. E. Katz y R. C. Haddon. Science 269, 1560 (1995)

6. J. L. Reddinger, G. A. Sotzing y J. R. Reynolds. Chem. Commun. 15, 1777 (1996).

7. “Langmuir Blodgett Films”, G. G. Roberts, Plenum Press, New York, 1990.

8. H. Muguruma, T. Saito, A. Hirotsuka y I. Karube. Langmuir 12, 5451 (1996).

9. S. Hotta y K. Waragai. J. Phys. Chem. 97[29] 7427 (1993)

10. V. Hernández, F. J. Ramírez, J. Casado, S. Hotta, G. Zotti y J. T. López Navarrete. J. Chem. Phys. 104, 9271-82 (1996); J. Casado, S. Hotta, H. Hernández y J. T. López Navarrete. J. Phys. Chem. A 103, 816 (1999); J. Casado, S. Hotta, H. Hernández y J. T. López Navarrete. J. Chem. Phys. 109[23] 1920-29 (1998).

11. J. T. López Navarrete y G. Zerbi. J. Chem. Phys. 94, 957 (1991); J. T. López Navarrete y G. Zerbi. J. Chem. Phys. 94, 965 (1991). 